\title{
Actions pédagogiques en nanosciences
}

\author{
H. Cazin d'Honincthun ${ }^{\mathrm{a}, \mathrm{b}}$
}

${ }^{a}$ Labex Nano-Saclay, Université Paris-Saclay, Saclay, France

${ }^{\mathrm{b}} \mathrm{C} 2 \mathrm{~N}$, Centre de Nanosciences et Nanotechnologies, Université Paris-Sud, Orsay, France

Contact email : hugues.cazin@u-psud.fr

Nous présentons ici le projet Nano-Ecole Ile-de-France de sensibilisation aux nanosciences et nanotechnologies à destination des écoles, des lycées et du grand public. Nous exposons le format et le détail du contenu des interventions réalisées au sein d'établissements scolaires. Enfin nous présentons la mise en place de formations proposées aux enseignants du secondaire ainsi que les actions à destination des élèves de primaire et du grand public.

\section{Le projet}

Nano-Ecole est à l'origine un projet national initié en 2010 sur 4 pôles (Grenoble, Lille, Paris-Saclay et Toulouse), visant à expérimenter l'introduction des nanotechnologies à l'école en associant enseignement scientifique et réflexion socio-scientifique dans le cadre du plan en faveur des nanotechnologies Nano-Innov.

Depuis, le projet Nano-Ecole Ile-de-France poursuit ces actions auprès des établissements scolaires et du public de la région Ile-de-France. Aujourd'hui, il est principalement soutenu par le Laboratoire d'Excellence en Nanosciences de Saclay (Labex Nano-Saclay), mais il est également soutenu par l'Université Paris-Sud, le Centre de Nanosciences et Nanotechnologies à Orsay (C2N), mais aussi par le CNFM et dans le cadre du projet IDEFI-FINMINA. Il a également reçu un soutien important du C'nano-IdF par le passé.

\section{Pédagogie du projet Nano-Ecole Ile-de-France}

Le projet pédagogique de Nano-Ecole Ile-de-France (1) repose en premier lieu sur l'interdisciplinarité que nous avons souhaité mettre en avant dans le contenu proposé. Afin d'augmenter l'attractivité du projet, nous avons choisi un format original de présentations théoriques vulgarisées et interactives où les élèves sont invités à participer activement, mêlées à des activités concrètes de mise en application par les élèves qui réalisent eux mêmes les expériences. Le contenu pédagogique a été développé de sorte que les grandes idées et concepts fondamentaux des nanosciences qui sont abordés dans ces interventions, se rapportent à des notions inscrites au programme scolaire officiel de l'éducation nationale. Dans le cadre de ce projet et pour certaines actions spécifiques, les élèves peuvent être mis en contact avec de gros instruments scientifiques de laboratoire notamment dans le cadre de visites de salles blanches et d'activités autour de la microscopie où le dialogue avec les chercheurs y est favorisé.

\section{Modalités des interventions}

L'initiation aux nanosciences commence dès le plus jeune âge avec un atelier à destination des 8-13 ans. Nous le proposons depuis deux ans dans le cadre de la Maison d'Initiation et de Sensibilisation aux Sciences (MISS) du projet Diagonale de Paris-Saclay 
(2) et de la région IdF, localisée sur le campus d'Orsay. Cet atelier d'une journée est mené par 4 doctorants et propose aux enfants une initiation aux nanosciences autour d'activités sur la thématique des couleurs.

Nous avons également développé des actions auprès des collégiens qui se déroulent principalement auprès des classes de $4{ }^{\text {ème }}$ car les notions exposées traitent encore une fois de la couleur qui est au programme. La première partie de l'atelier est une introduction interactive d'une heure aux dimensions, aux échelles et aux propriétés autour d'expériences interactives et de vidéos. La seconde partie est une séance d'une demi-journée se déroulant en laboratoire ou au sein de l'établissement et s'articule autour d'ateliers sur la synthèse des couleurs, la composition de la lumière et sur les couleurs du nanomonde.

Les interventions avec les classes de lycées constituent la grande majorité de nos actions et cela concerne plusieurs centaines d'élèves de la région IdF chaque année.

L'intervention auprès de chaque établissement se déroule sur plusieurs séances afin de construire un cheminement dans la transmission des concepts fondamentaux des nanosciences. Cela se déroule le plus souvent sur 2 ou 3 séances de 60 à 120 minutes chacune et qui associent des exposés et des expériences que les jeunes réalisent eux-mêmes. Le format est donc variable et adaptable selon les attentes de l'enseignant, des élèves ou de l'établissement et en fonction des sujets traités. La première séance se déroule au sein de l'établissement scolaire et consiste en une conférence interactive au cours de laquelle les concepts de bases sont exposés. Pour la seconde séance, si l'établissement scolaire le permet, ce sont les élèves qui viennent au sein d'un laboratoire de recherche afin d'y rencontrer des chercheurs et visiter les salles d'expériences. La dernière séance, qui se déroule en établissement scolaire, propose, dans la continuité des séances précédentes, de mettre en évidence des principes fondamentaux des nanosciences sur les heures de Travaux Pratiques et donc en demi-classes.

Comme nous le verrons par la suite, la mise en application de ces notions se fait, selon le choix de l'enseignant, au travers d'un TP de synthèse de nanoparticules d'or réalisée en binôme, autour d'un TP sur les couleurs structurelles au travers des papillons morphos, naturellement nanostructurés, ou sur un TP microscopie, allant du microscope optique au microscope à effet tunnel en passant par la microscopie électronique à balayage.

\section{Quelques chiffres}

Chaque année nous intervenons auprès de plusieurs centaines de lycéens et collégiens et dans de nombreuses manifestations scientifiques. Le tableau 1 présente des statistiques du projet sur les 4 dernières années sachant que le projet existe depuis 6 ans.

TABLEAU I. Chiffres concernant les interventions Nano-Ecole IdF.

\begin{tabular}{ccccc}
\hline Années & Primaires & Collégiens & Lycéens & Enseignants \\
\hline Effectifs & 870 & 410 & 2368 & 152 \\
Etablissements différents & 18 & 6 & 24 & 14 \\
Nombre d'heures cumulées & 191 & 65 & 305 & 80 \\
Nombre de classes & 29 & 14 & 97 & $/$ \\
\hline
\end{tabular}

\section{Les démonstrateurs}

L'originalité du projet repose en partie sur des nano-démonstrateurs portables, qui se présentent sous forme de mallettes dont certaines ont été financées en partie dans le cadre de l'IDEFI-FINMINA ou d'appels à projet CNFM. L'objectif est d'avoir des démonstrations mobiles pour permettre aux jeunes et au grand public de se familiariser 
avec les nanosciences et leurs applications et d'en illustrer quelques principes fondamentaux.

Le contenu matériel et pédagogique de ces mallettes est adaptable suivant les conditions d'utilisations et le public visé. Même si elles sont principalement utilisées dans le cadre des actions Nano-Ecole, elles sont prêtes à l'emploi afin de permettre à tout scientifique désireux de faire de la diffusion scientifique de s'approprier rapidement les contenus pour des actions grand public ou en milieu scolaire. Une mallette est donc composée de l'exposé que l'intervenant est libre d'adapter à son public, des fiches explicatives et bien entendu du matériel.

\section{Mallette : Comprendre les nanos}

Nous proposons un premier module qui traite des généralités sur les nanosciences et les nanotechnologies et en expose les bases. Il est utilisé dans le cadre des conférences et de toutes les séances introductives aux nanos.

Des études montrent que les jeunes rencontrent des difficultés à appréhender les petites échelles (3). Nous abordons la notion d'échelle sous la forme d'un quizz réalisé à partir de boitiers de vote de type auto-école. Les élèves doivent classer des objets connus ou inconnus, visibles ou invisibles sur une échelle graduée. Cela a l'avantage d'être à la fois ludique et de rendre l'apprentissage plus concret et de découvrir quelques objets tels que le nanotube de carbone ou le transistor.

Le reste du kit permet d'aborder quelques concepts de base des nanosciences, aussi connus sous le nom de «Big Ideas » (3) en complément de séquences vidéo sur les nanosciences judicieusement choisies. La mallette contient tout le matériel pour mettre en avant le comportement des liquides magnétiques, les ferrofluides. Autre exemple, on y trouve une expérience autour de la poudre de spores de Lycopode qui a la particularité de générer de grandes flammes lorsqu'elle est dispersée dans l'air et donc d'illustrer les notions de rapport surface sur volume et de réactivité.

La dernière partie du module propose une sorte de comparatif entre les grands domaines applicatifs des nanotechnologies, les applications qui devraient permettre une amélioration de la qualité de vie dans les prochaines années, et des applications potentiellement à risque si leur impact sur l'environnement où la santé, aujourd'hui à l'étude, est un jour démontré. Cette approche a pour objectif d'illustrer la notion de rapport bénéfice-risque qui est pertinente dans le cadre de l'évaluation des risques industriels et qui est ensuite discutée avec les élèves.
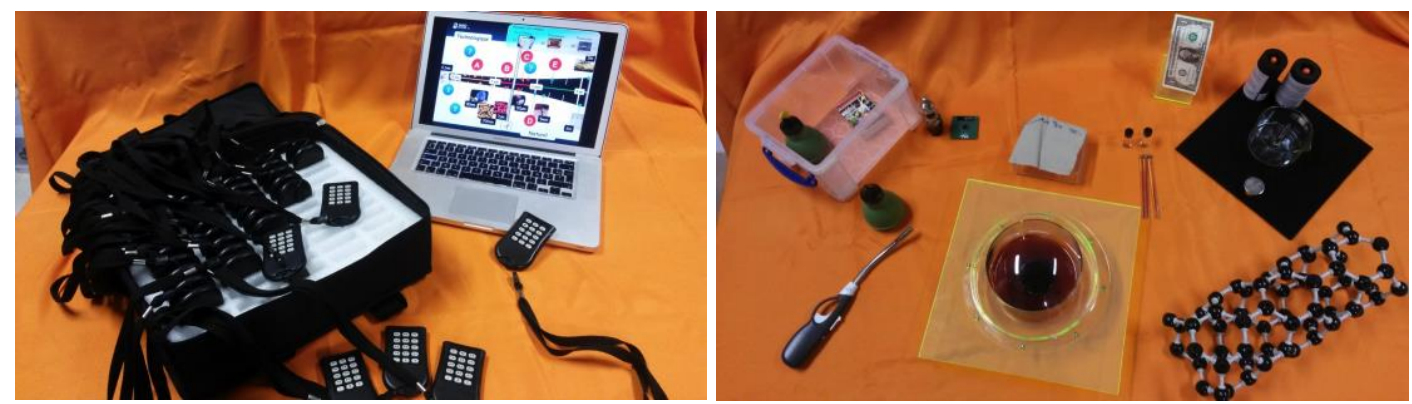

Fig.1. Contenu de la mallette « Comprendre les nanos ». A gauche, on retrouve les boitiers de vote utilisés pour le quizz. L'image de droite présente les différentes expériences et objets de démonstration de ce module : ferrofluides, lycopodes, le nanotube de carbone, les processeurs, les tissus hydrophobes. 


\section{Malette : Nanoparticules d'or}

Ce module a pour objectif de mettre en évidence le changement de propriétés de la matière aux dimensions nanométriques en étudiant les propriétés optiques de ce matériau fascinant qu'est l'or. On part des connaissances des élèves sur les propriétés de ce matériau pour les amener à constater par eux-mêmes que les propriétés optiques du matériau changent à l'échelle nanoscopique. Le travail expérimental est assez simple et consiste en une synthèse de nanoparticules d'or monodisperses par voie aqueuse, formalisée en 1951 par J. Turkevich (4) à partir du matériel de chimie de Lycée usuel (plaque chauffante, béchers, erlenmeyers, pipettes graduées) et d'une fiche de TP. Cette réaction consiste en une réduction de sels d'or (acide tétrachloroaurique de formule $\mathrm{HAuCl}_{4}$ ) par du citrate de sodium en excès $\left(\mathrm{Na}_{3} \mathrm{C}_{6} \mathrm{H}_{5} \mathrm{O}_{7}\right)$ qui joue également le rôle de stabilisant. Précisons que les réactifs utilisés sont fortement dilués et ne présentent donc pas de risques majeurs pour la sécurité.
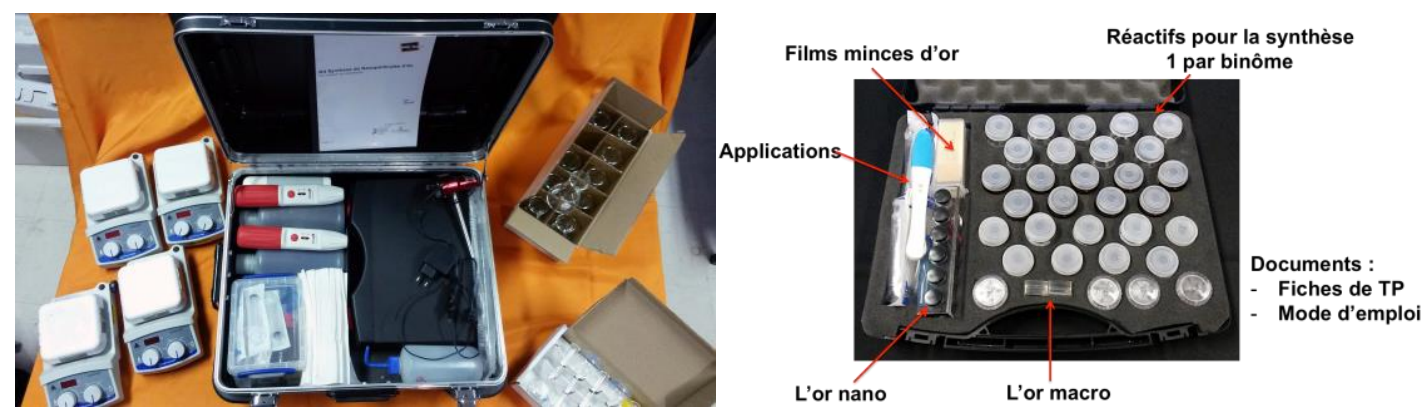

Fig.2. Contenu de la mallette «synthèse de nanoparticules d'or ». Elle contient tout le matériel, les fiches, les manuels et la verrerie pour réaliser les expériences auprès de 20 personnes en binôme sur des sessions de 60 à 90 minutes. A droite on retrouve la mallette « réduite » contenant les réactifs et le matériel nécessaire à la présentation en lycée où le matériel est déjà présent.

L'élève effectue une étape de dilution, met la solution à chauffer et obtient après ajout du citrate en quelques minutes une solution collö̈dale constituée de nanoparticules d'or de $20 \mathrm{~nm}$ de couleur rouge rubis. On peut montrer qu'en modifiant le ratio sels d'or sur citrate la taille des nanoparticules change et donc la couleur de la solution colloïdale obtenue. Le phénomène à l'origine de cette couleur correspond à un effet de résonnance de plasmons de surface localisés dans chaque nanoparticule d'or. Le kit contient des nanoparticules d'or en bâtonnets (nanorods), synthétisées au Laboratoire de Chimie Physique d'Orsay, illustrent que la couleur de ces nano-objets est fortement dépendante de la géométrie de la particule. A partir de la solution obtenue, l'ajout de gouttes de $\mathrm{NaCl}$ perturbe la stabilisation électrostatique des nanoparticules et la couleur change. Les différentes étapes du TP sont présentées dans la vidéo TP-or.

Tout au long du TP, l'élève reprend diverses notions du programme telles que la dilution, la conservation de la quantité de matière, l'oxydo-réduction.

Dans la dernière partie de la séance, des exemples d'applications des nanoparticules d'or sont présentés tels que le test de grossesse ou des applications plus générales dans les domaines de la biologie et du biomédicale tels que les capteurs biologiques, les tests rapides, etc.. On aborde également l'utilisation des nanoparticules d'or pour la localisation de tumeurs et le traitement du cancer par hyperthermie ou l'utilisation très rependue de nanoparticules métalliques dans le domaine de l'art. 
Pour terminer, on propose de revenir à un état micro-macroscopique de l'or en filtrant la solution et l'élève repart à l'issue de la séance avec un joli film d'or.

Nous avons adapté le contenu de cette mallette en proposant de synthétiser des nanoparticules et d'en expérimenter les propriétés et l'avons rendu commercialisable. Elle est actuellement proposée (5) par la société Jeulin, éditeur de solutions pédagogiques pour l'enseignement scientifique et technique.

\section{$\underline{\text { Malette : Couleurs structurelles }}$}

Ce module traite des couleurs structurelles, largement présentes dans la nature avec pour exemples la plume de paon, l'opale, les papillons morpho bleus ou un objet artificiel tel que le CDROM, dont les couleurs sont directement liées à l'organisation de la matière. On constate un phénomène de changement de couleurs suivant la manière dont on regarde l'objet, que l'on appelle : l'iridescence (6). Ce sujet fait appel à des notions abordées en première scientifique telles que le spectre électromagnétique, l'optique, la dispersion et des notions de terminale telles que les propriétés des ondes, les phénomènes de diffraction et d'interférences.

L'exposé et le matériel présent dans cette mallette proposent d'observer à différentes échelles la structure d'un papillon morpho bleu en utilisant des microscopes optiques ou des images prises au microscope électronique à balayage de même que des maquettes ont également été réalisées pour appuyer ces observations. Le kit propose également de comprendre les phénomènes ondulatoires observés au travers de l'étude de structures proches que sont le CD, le DVD et le Blu-Ray. Enfin, des maquettes et expériences inspirées par des travaux de chercheurs de l'Institut des NanoSciences de Paris (INSP) et de l'Institut d'Optique Graduate School finissent de convaincre de la différence entre couleur pigmentaire et structurelle et donc de la relation entre la structuration et la couleur observée.

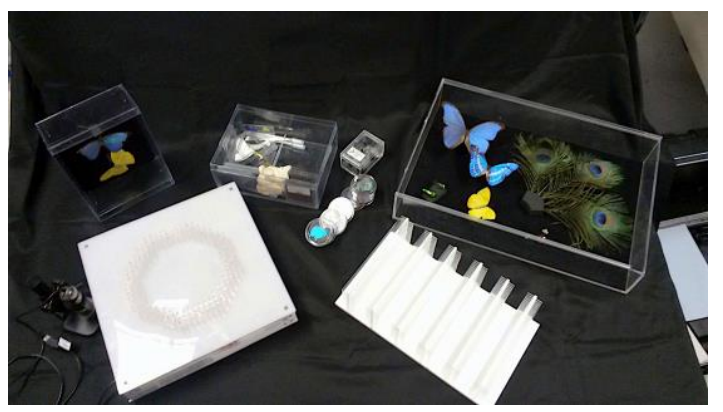

Fig.3. Contenu de la mallette sur les couleurs structurelles. Elle est composée de vitrines présentant les différents objets iridescents, des maquettes d'ailes de papillon ou de structures photoniques, des microscopes USB et tout le matériel nécessaire pour réaliser le TP en Lycée. En bonus, la vidéo de démonstration montre que les couleurs sont structurelles en vaporisant de l'alcool à la surface de l'aile qui change de couleur.

\section{Malette : Microscopie}

L'idée de ce module est de proposer une descente progressive vers l'infiniment petit par une présentation synthétique et interactive. Le principe est de partir de l'œil et de ce qu'il est possible d'observer à cette échelle, pour passer à l'étude du microscope optique. Pour cela, nous avons fait l'acquisition de microscopes en réflexion USB Dino-lite qui sont dotés de grossissements x 20-70 et x500 et d'accessoires divers dont des filtres polariseurs. Par 
facilité et soucis de cohérence avec la séance sur les couleurs structurelles, des ailes de papillons sont notamment observées. Elles présentent une structure en écaille de 50 microns par 100 microns.

Pour permettre de descendre un peu plus loin dans la matière, nous utilisons un microscope électronique à balayage portable TM3000 de marque Hitachi, financé grâce au soutien de Nano-Innov, du C'nano-Idf et du CNFM. Cet appareil transportable peut être facilement utilisé en salle de classe par les élèves. Les élèves en quart de groupes, sont invités à utiliser eux mêmes le microscope et à réaliser un ensemble d'observations et de mesures sur les structures des ailes de papillons observées qui sont à l'échelle nanométrique.

Afin de n'avoir à gérer que des groupes réduits, des observations en microscopie champ proche sont réalisées en parallèle. Nous offrons la possibilité d'utiliser un microscope à force atomique et d'un microscope à effet tunnel NaioSTM® de marque Nanosurf que nous avons acquis tout récemment. Parmi les échantillons utilisés avec 1'AFM, on propose le DVD pour lequel les élèves sont invités à observer et mesurer la largeur des pistes. Ces observations permettent de mettre en évidence l'intérêt du microscope en tant qu'outil essentiel de recherche permettant de comprendre des phénomènes et de mesurer des objets de l'infiniment petit.

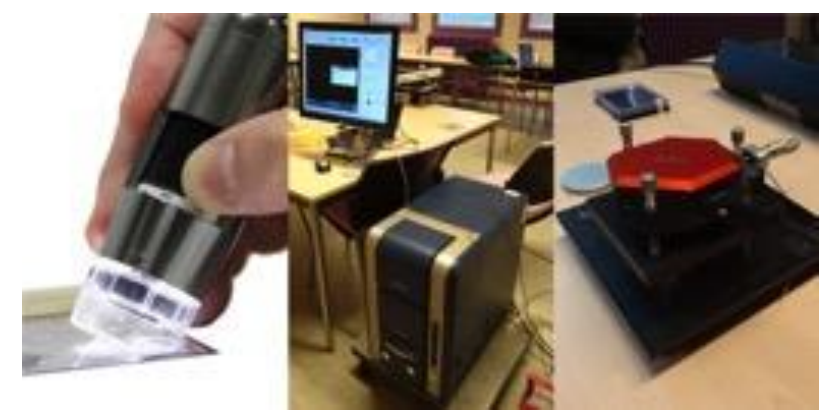

Fig.4. Kit microscopie. Il est composé de microscopes optiques USB, d'un microscope électronique à balayage, d'un microscope à force atomique et d'un microscope à effet tunnel.

\section{Malette : Dépollution au dioxyde de Titane $\left(\mathrm{TiO}_{2}\right)$}

Nous avons réalisé avec un laboratoire partenaire (LCP d'Orsay) une mallette mettant en avant la dépollution à partir de nanoparticules de dioxyde de titane. Ce module permet d'aborder des concepts tels que le rapport surface sur volume favorable aux nanos, les nanoparticules, l'énergie et la recherche. Elle est composée d'un réacteur de photocatalyse basé sur un substrat de mousse Quartzel® PCO photocatalytique (Saint-Gobain) contenant des nanoparticules de dioxyde de titane, enfermé dans une enceinte étanche en verre et soumis à la radiation d'une lampe UV au centre du réacteur. Une pompe péristaltique permet la circulation d'une solution polluée par un colorant organique. On constate la dégradation du colorant par le $\mathrm{TiO}_{2}$ sous l'action des $\mathrm{UV}$. Un réacteur témoin ne contenant pas de mousse photocatalytique permet de valider le résultat. 


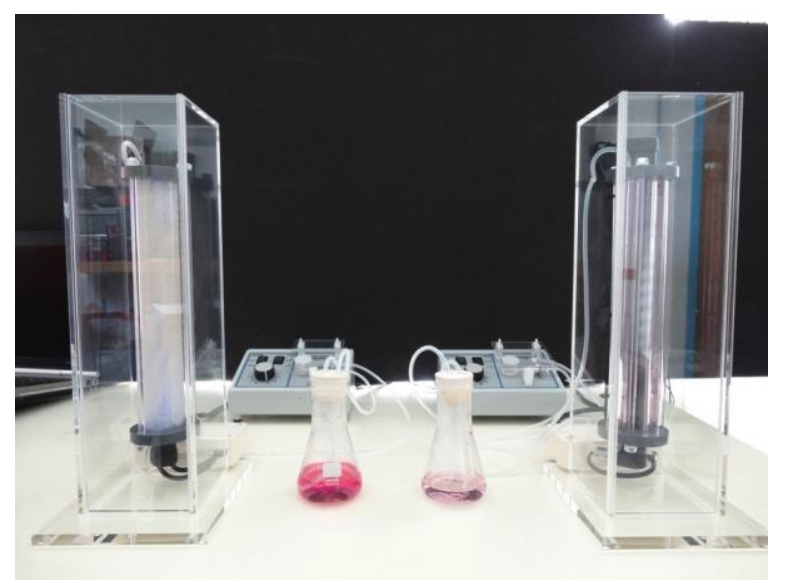

Fig.5. Kit sur la photocatalyse à base de nanoparticules de dioxyde de titane $\left(\mathrm{TiO}_{2}\right)$. Il comprend un réacteur de photocatalyse et un réacteur témoin, deux pompes péristaltiques, tout les accessoires nécessaires au fonctionnement des réacteurs et à la démonstration du procédé.

\section{III.La formation}

\section{Formation des enseignants}

Depuis 5 ans, nous proposons chaque année une formation de 2 jours à une trentaine d'enseignants du second degré de la région IdF. Cette action est menée dans le cadre du Plan Académique de Formation des enseignants du secondaire. A l'occasion de cette formation, des chercheurs et enseignants chercheurs de l'Université Paris-Saclay leur présentent les grands concepts et les grandes avancées de la recherche en nanosciences et nanotechnologies par une approche interdisciplinaire allant de la Physique jusqu'à la Chimie en passant par les Nanobiotechnologies. On propose également des ateliers pratiques et des activités autour de la salle blanche et des techniques de microscopie. Enfin, nous échangeons avec eux et leur proposons des pistes afin qu'ils puissent aborder les nanosciences au sein de leurs classes.

\section{Formation en ligne}

Le projet Nano-Ecole a participé à la conception d'un MOOC (Massive Open Online Course), un cours en ligne gratuit et ouvert à tous de 7 semaines pour «Comprendre les nanosciences » (7) diffusé sur la plateforme France Université Numérique. On y retrouve 50 enseignants-chercheurs et chercheurs de 1'Université Paris-Saclay qui font découvrir au travers de 80 vidéos de cours ce vaste domaine en allant de la Physique et de la Chimie jusqu'aux Biotechnologies et à la Médecine, sans oublier les enjeux sociétaux, sanitaires et environnementaux.

\section{IV.Conclusion}

Alors que le projet Nano-Ecole IdF existe depuis maintenant 6 ans, il a atteint une certaine maturité et une certaine reconnaissance auprès des établissements de la région. De nouvelles demandes s'ajoutent chaque année aux demandes récurrentes, ce qui permet au projet de toucher différents publics, différents niveaux, d'étendre l'action et d'intervenir partout en Ile-de-France. Une des forces du projet est sa faculté à s'adapter et à répondre aux différentes demandes des enseignants pour s'implanter au mieux au sein de la progression pédagogique de la classe. Au fil des ans, les contenus et les manips ont été optimisés afin de répondre totalement aux attentes des établissements. De nouvelles manips 
sont en développement et devraient permettre de renouveler l'offre de l'action Nano-Ecole IdF.

\section{Remerciements}

Nous remercions tout particulièrement le Labex Nano-Saclay (8) pour le fort soutien au projet. Nous remercions également le soutien du GIP-CNFM (10) et du projet IDEFIFINMINA (9) dans le cadre du programme Grands Investissements d'Avenir (PIA1).

\section{Références}

1. Nano-Ecole Ile-de-France. Website: http:// www.nano-ecole.fr/idf

2. MISS : Maison d'Initiation et de Sensibilisation aux Sciences du projet Diagonale Paris-Saclay et de la région Ile-de-France. Website : http://hebergement.u-psud.fr/miss/

3. B. Hingant, V. Albe, "Nanosciences and nanotechnologies learning and teaching in secondary education: a review of literature", Studies in Science Education, Vol. 46, 2 (2010), DOI 10.1080/03057267.201.504543.

4. J. Turkevich, P. C. Stevenson, J. Hillier, "A study of the nucleation and growth processes in the synthesis of colloidal gold", Discuss. Faraday. Soc., 11, 55-75 (1951

5. Kit Nanoparticules vendu chez Jeulin. Website: http://www.jeulin.fr/tp-synthese-de-nanoparticulesd-or-ress-tp00310.html

6. S. Berthier, E. Charron, J. Boulenguez, "Morphological structure and optical properties of the wings of Morphi-dae", Insect Science, Volume 13, Issue 2, pages 145-158, (2006).

7. MOOC «Comprendre les nanosciences»: website: https://www.funmooc.fr/courses/UPSUD/42003/session01/about

8. LABEX NANO-SACLAY : Laboratoire d'Excellence en Nanosciences de Saclay. Website: http://nanosaclay. $f r /$

9. IDEFI-FINMINA : Initiative d'Excellence - Formation Innovante en MIcroélectronique et Nanotechnologies, ANR-11-IDFI-0017. Website: http://www.cnfm.fr/VersionFrancaise/actualites/ FINMINA.htm

10. GIP-CNFM: Groupement d'Intérêt Public - Coordination Nationale pour la formation en Microélectronique et en nanotechnologies. Website: http://wwww.cnfm.fr 